\title{
Germanica
}

\section{Polnische Migranten in Deutschland, deutsche Minderheit in Polen - zwischen den Sprachen und Kulturen}

\section{Elżbieta Katarzyna Dzikowska}

\section{CpenEdition}

\section{Journals}

Édition électronique

URL : http://journals.openedition.org/germanica/422

DOI : 10.4000/germanica.422

ISSN : 2107-0784

Éditeur

Université de Lille

\section{Édition imprimée}

Date de publication : 1 juin 2006

Pagination : 11-24

ISBN : 2-913857-17-5

ISSN : 0984-2632

Référence électronique

Elżbieta Katarzyna Dzikowska, «Polnische Migranten in Deutschland, deutsche Minderheit in Polen zwischen den Sprachen und Kulturen », Germanica [Online], 38| 2006, Online erschienen am: 19

Februar 2010, abgerufen am 06 Oktober 2020. URL : http://journals.openedition.org/germanica/422 DOI : https://doi.org/10.4000/germanica.422

Ce document a été généré automatiquement le 6 octobre 2020.

(c) Tous droits réservés 


\title{
Polnische Migranten in
} Deutschland, deutsche Minderheit in Polen - zwischen den Sprachen und Kulturen

\author{
Elżbieta Katarzyna Dzikowska
}

1 In dem von Carmine Chielino 2000 [CHIELLINO:2000] herausgegebenen, über 400 Seiten starken Handbuch zur interkulturellen Literatur in Deutschland werden Polen im Kapitel „Literatur osteuropäischer Migrant/innen“ anderthalb Seiten gewidmet. Die bereits im Titel aufgeführte Hypothese - „Polen, ein Sonderfall?“ - wurde zusätzlich mit einem Fragezeichen versehen, was deren Widersprüchlichkeit in bezug auf die einführende Bemerkung etwas einschränkt, nach der die jüngsten Migrationswellen aus osteuropäischen Ländern eng mit Entwicklungen in der ehemaligen Sowjetunion verknüpft seien. So situiert sich auch dieser in vielem sehr ungenau recherchierte Bericht im dicht besetzten Umfeld einer verkürzten, weil stark stereotypisierten Wahrnehmung, oder besser gesagt, Nichtwahrnehmung Ostmitteleuropas.

2 Obwohl im Kapitel 7. die Literatur der Russlanddeutschen und im Kapitel 8. die der russischen Emigrant/innen bereits thematisiert wurde, kehrt der Autor des osteuropäischen Kapitels, Klaus-Peter Walter, gleich am Anfang wie auch im ersten Unterkapitel, betitelt: „Sprache, Themen und Motive der Exilliteratur“ erneut und fast ausschliesslich auf Autoren aus der ehemaligen Sowjetunion zurück, um in die Besonderheiten der deutschsprachigen Literatur von Migrant/innen aus dem tschechischen, slowakischen, polnischen, bulgarischen oder ungarischen Kulturkreis einzuführen. Dadurch bekommen wichtige Faktoren einen anderen Stellenwert oder sie müssen sogar von der Bildfläche verschwinden, wie die zentrale Frage, welche Funktion dem Deutschen als einer Kommunikatationssprache in Ostmitteleuropa, also in einem über die deutsche Staatlichkeit hinausgehenden Wirkungsbereich, historisch zukam. 
3 Mögliche Folgen einer solchen Reduktion möchte ich an den im Handbuch behandelten Beispielen kurz aufzeigen. Im polnischen Unterkapitel wurde der $1928 \mathrm{im}$ polnischen Bochnia geborene Satiriker, Gabriel Laub, als einziger Autor fiktionaler Prosa in einem Absatz kurz besprochen. Neben ihm widmete der Autor seine flüchtige Aufmerksamkeit dem Deutschen Polen-Institut in Darmstadt und erwähnte dabei den „ausgezeichneten Übersetzer“ Karl Dedecius samt dessen „zahllosen Freundschaften“ mit polnischen Autor/innen, die ihm ihre "internationale Bekanntheit" und im Fall Wisława Szymborska sogar den Nobelpreis zu verdanken haben. Hauptsächlich konzentrierte er sich aber auf die in seiner Auffassung umstrittene Rolle des „wohl bekanntesten Literaten der polnischen Herkunft", Marcel Reich-Ranicki, für die deutsche Öffentlichkeit. Dessen „unbändiger Drang zur Selbstdarstellung“, seine starke Medienpräsenz, das charakteristische näselnde Lispeln blieben nicht unerwähnt, mit keinem Wort dagegen würdigte Klaus-Peter Walter die für die Formung der multikulturellen Identität des einflussreichen Kritikers historisch wichtigen Kontexte. Zum Abschluss seiner kurzen Besprechung des Sonderfalls Polen erwähnte er noch den „Romancier und Journalisten“ Tadeusz Nowakowski, der seine hauptsächlich der polnischen Literatur, darunter Stanisław Lem, gewidmeten Kritiken für die FAZ auf deutsch, seine Romane dagegen auf polnisch schrieb.

Zieht man die von Klaus-Peter Walter völlig ausgesparten politischen Kontexte und biographischen Hintergründe heran, unter deren Einwirkung die von ihm genannten Autoren in einem bestimmten Abschnitt ihres Lebens den Entschluss fassten, in den deutschsprachigen Raum zu gehen, meistens als Exulanten, um dort unterschiedliche Wege der Akkulturation/Assimilation einzuschlagen, so wird schnell deutlich, dass der Sammelbegriff „polnische Migrantenliteratur in Deutschland“ auf recht heterogäne Untersuchungsgegenstände hindeutet und komplexe Prozesse eines Kulturaustauches von langer historischer Dauer mit einschliesst. Es zeigt sich daher von Nutzen, literarhistorische Prozesse in beiden Gesellschaften, der polnischen wie der deutschen, im Auge zu behalten, will man die polnisch-deutsche Transkulturalität in all ihren Facetten gründlich und fern von ideologisch vorgeprägten Setzungen erfassen.

5 Als Gabriel Laub 1928 in der Nähe von Krakau geboren wurde, lag seine westgalizische Geburtstadt Bochnia seit 10 Jahren erneut in den Grenzen des 1918, nach 125 Jahren der Teilung, wieder errichteten polnischen Staates. Die polnisch-jüdische Kaufmannsfamilie floh 1939, nach dem Ausbruch des Zweiten Weltkrieges, nach Lemberg, und wurde von den Sowjets, die diese polnische Stadt auf Grund des Paktes Ribbentropp-Molotow 1939 besetzten, sehr schnell nach Sibirien deportiert. Gabriel Laub überlebte, kehrte 1945 nach Krakau zurück, bestand dort 1946 das Abitur und entschloss sich, nach dem Politologie-Studium in Prag, nicht mehr nach Polen zurück zu kehren, sondern in der Tschechoslowakei zu bleiben.

6 Bochnia, Krakau, Lemberg und Prag lagen bis 1918 in den Grenzen der HabsburgerMonarchie. Seit dem Mittelalter, als sich Bochnia, Krakau und Lemberg noch innerhalb des polnischen Staates und Prag im Heiligen Römischen Reich Deutscher Nation befanden, fungierte Deutsch in jenen Städten als eine wichtige Kommunikationssprache. Stark vertreten war dort die mosaische Glaubensgemeinschaft, und es fehlte auch nicht an deutschen Menschen.

7 Der Journalist Laub musste 1968 Tschechien in Folge des Prager Frühlings verlassen, und er ging zunächst nach Wien, dann nach München und Hamburg, wo er 1998 verstarb und später in Tel Aviv beigesetzt wurde. Sein literarisches Werk, Aphorismen 
und Satiren, abgesehen von seinem in Tschechisch verfassten Buchdebüt „Skusenosti“ [LAUB:1967] verfasste er hauptsächlich in Deutsch. Er übersetzte ins Deutsche Autoren, welche sich der kommunistischen Diktatur widersetzten, wie Havel, Kołakowski und Sołżenicyn. Seine von der politischen Verfolgung jeglicher Coleur gezeichnete Vita wurde in dem von Carmine Chielino herausgebrachten Handbuch auf folgende Kurzformel zurückgebracht: „Er war schon vor seiner Niederlassung im Westen 1968 ein Wanderer zwischen den Sprachwelten (polnisch, tschechisch und russisch) und bezeichnete sich selbst als „Pole von Geburt, Tscheche aus Neigung und Weltbürger ohne Pass“" [CHIELLINO: S.195].

Ebenfalls in einem polnisch-jüdischen Millieu, ebenfalls in einer Kaufmannsfamilie, allerdings in einem westlichen Winkel Polens, der in Folge der Teilungen an Preussen gefallen war, kam 1920, als der wieder errichtete polnische Staat erst zwei Jahre alt war, Marcel Reich-Ranicki zur Welt. Die Familie emigrierte 1929 nach Deutschland, wo Reich-Ranicki in Berlin ein Gymnasium besuchte und von einem Studium in Deutschland träumte. 1938 haben die Nazis polnische Staatsangehörige jüdischer Abstammung nach Polen zwangsdeportiert. Die polnischen Behörden wollten aber den polnischen Juden aus Deutschland den Einlass zunächst nicht gewähren. So mussten die gedemütigten Menschen an der deutsch-polnischen Grenze längere Zeit verbringen.

9 Auf solchem Weg kamen die Reichs nach Warschau. Marcel Reich-Ranicki konnte die tragische Zeit der deutschen Besatzung im Warschauer Ghetto und später in einem Versteck auf der sog. arischen Seite überleben. 1945 kehrte er als offizier der kommunistischen Armee nach Warschau zurück. Bald wurde er im Partei-Organ „Trybuna Ludu“ zu einem führenden, der Lukacs' Doktrin stark verpflichteten Kritiker.

Die Beschäftigung mit der Literatur setzte er nach seiner 1958 erfolgten Ausreise in die BRD fort. Deutsch war seit seiner Kindheit im grosspolnischen Włocławek eine wichtige Kommunikations- und Kultursprache für ihn, und eine verhasste Feindessprache für seine polnische Umgebung. In der Beschäftigung mit den in dieser Sprache verfassten sprachlichen Kunstwerken sah Reich-Ranicki eine Grundlage für eine solche Identifikation mit Deutschland, welche ohne Aufgabe seiner jüdisch-polnischen Herkunft herzustellen war.

Die Vielvölkerstadt Łódź, welche erst im 19. Jh., in Folge einer raschen Industrialisierung, zu einer eher hässlichen Grosstadt im Zarenreich avancierte, wurde 1921 Geburtsort des Deutschen Karl Dedecius. Nach dem Ersten Weltkrieg wurde die Stadt wieder polnisch. 1939 bestand Karl Dedecius in einem polnischen Gymnasium das Abitur, und wurde bald darauf zur deutschen Wehrmacht eingezogen, weil die Nazis das polnische Land sehr schnell besetzten. Als deutscher Soldat kam er in die russische Kriegsgefangenschaft und von dort kehrte er, mit einem Zwichenaufenthalt in Weimar, in die BRD zurück. Als Beamter der Ver-sicherungsgesellschaft „Allianz“ in Frankfurt hat er seit den 50er Jahren sein imposantes Lebenswerk ohne jegliche staatliche Förderung, als eine Initiative von ganz unten angefangen. Allmählich wurde Dedecius zu der wichtigsten Institution im deutsch-polnischen Kulturaustausch. Derzeit fällt diese Funktion dem von ihm gegründeten Deutsch-Polnischen Institut in Darmstadt zu. Innerhalb seiner Übersetzungsarbeiten nahmen die Aphorismen des Lemberger Meisters von Gabriel Laub, Stanisław Jerzy Lec, einen bedeutenden Platz ein. Dedecius trug wesentlich dazu bei, dass „Die unfrisierten Gedanken“ von Stanisław Jerzy Lec, einem polnischen Autor, der ebenfalls manche Texte in Deutsch verfasste, weltbekannt wurden. Es wäre vielleicht eine Untersuchung wert, sprachliche Formulierungen für 
Aphorismen im Dreieck: Lec, Laub und Dedecius, zwischen Polnisch, Deutsch und Tschechisch, mit einander zu vergleichen.

13 Tadeusz Nowakowski war Sohn eines 1889 geborenen Journalisten und Schriftstellers, Stanisław Nowakowski, der sich in Ermland und Masuren in den politischen Auseinandersetzungen zwischen Polen und Deutschen als glühender polnischer Patriot sehr stark engagierte. Nach dem Ersten Weltkrieg wurde er Mitglied des PlebiszitAusschusses in Warschau, und organisierte den Bund der Polen in Ermland und Masuren. Die Nazis brachten ihn bereits im September 1939 ins KL Dachau, wo er 1942 schliesslich ermordet wurde. Auch dem Sohn, der 1938 die Warschauer Polonistik absolvierte, wurde ein Aufenthalt in Konzentrationlagern der Nazis nicht erspart. Er überlebte und wurde die sog. displaced person. Bis 1947 verweilte er in einem Übergangslager an der deutsch-niederländischen Grenze und gab dort u.a. Polnischunterricht. Später lebte er als Mitarbeiter des BBC und des Senders Freies Europa zunächst in London, dann ab 1953 in München, schrieb darüber hinaus für die beiden wichtigsten Exilzeitschriften der Polen: für die Londoner „Wiadomości“ und die Pariser „Kultura“, und verfasste Romane. Mit der Zeit engagierte er sich immer mehr in den Kulturtransfer zwischen Polen und Deutschen. Als Romanautor wollte er sich allerdings ausschliesslich in der polnischen Sprache ausdrücken.

vier Autoren gehörten einer Generation an, die nach dem Ersten Weltkrieg zur Welt kam, und in dem neu errichteten polnischen Staat ihre nationalen Identifikationen in vielfachen Mischräumen zu treffen hatte, in welchen Deutsch eine noch lebendige Kommunikationssprache war. Sie rieben sich an aggressiven Nationalismen unterschiedlicher Prägung. Ihre ersten Erfahrungen mit der deutschen Sprache machten sie bereits in ihrer frühen Kindheit, die von einer Sprachenvielfalt noch geprägt war. Nach dem Zweiten Weltkrieg wurden all jene Räume zwangsmonoethnisiert.

Im Folgenden werde ich versuchen, in einem kurzen geschichtlichen Exkurs auf einige Besonderheiten der Nationsbildung in Polen hinzuweisen, welche für die Migrationsprozesse nach 1945 wichtig waren. Insbesondere wird mich die Frage beschäftigen, ob Polen ein Sonderfall dadurch wäre, weil es in Deutschland trotz einer Millionen starken Migration aus diesem Land so gut wie keine erwähnungswürdige polnische Migrantenliter aus der Feder der jüngeren Generationen gäbe, oder der Sonderfall Polen beruht eher darauf, dass die für die Erfassung des Problems angewandten Begriffe ihren Gegenstand manchmal verfehlen. Jedenfalls ergibt sich aus den ersten bibliographischen Recherchen eine Liste von immerhin ca 200 Namen der im Literaturbetrieb aktiv wirkenden Menschen, Wanderer zwischen den beiden Sprachwelten. [KALCZYŃSKA: 2001]

16 "Lass mich mahlen, und ruhe Dich aus“ - „daj u ja pobruszE, a ty pocziwaj“ - lautet der älteste, in einem Kloster im nćiederschlesischen Heinrichau aufgeschriebene Satz der polnischen Sprache. Lange Zeit galt er im kommunistischen Polen als ein wichtiger Beweis für das Polentum der nach 1945 „wiedergewonnenen“, vorhin deutschen, Gebiete im Westen und Norden des Landes. Die Nationalisierung des Blicks auf das Mittelalter, das, ähnlich wie die Antike, noch keine Nationen im modernen Sinn kannte, lag in der Aussparung des Kontextes.

17 Der oben angeführte Satz ist ein beredtes Zeugnis dafür. Er wurde nämlich von einem böhmischen Bauern geäussert, der sich zu seiner polnischen Frau in deren Muttersprache wandte. Seine Mitteilung, weil sie juristisch relevant war, wurde dann 
von einem deutschen Mönch in dessen in der lateinischen Sprache verfasste Chronik aufgenommen.

Es war im damaligen Niederschlesien, wie auch im ersten polnischen Staat, keine untypische Kommunikationssituation. Res publica Polonia, eine multiethnische Adelsrepublik, die in der Tradition des alteuropäischen Ständewesens stand und ein Vertragskönigtum war, blieb nämlich der ethnischen Zusammensetzung ihrer Bürger gegenüber als Staat neutral. Pole im Sinne „der polnische Bürger“, war, wer dem Adel gehörte, und der Adel war zahlreich (zwischen 10 und $40 \%$ aller Bürger in manchen Gegenden), sozial ungleich und multiethnisch. Der Begriff „Pole“ war somit ursprünglich kein ethnisch besetzter Begriff, ethnische Polen, wie etwa die Adligen Masowiens, wurden „koroniarze“, „Bewohner der Kronländer“ genannt, im Gegensatz etwa zu den Bewohnern Litauens. Auch die Bezeichnung „Litauen“ in dem Namen „Königreich Polen-Litauen“ war nicht ethnisch besetzt und bezog sich auch auf alle Bewohner östlich des Bug. Die nationale Identifikation beruhte im Königreich PolenLitauen auf Loyalität gegenüber dem König und dem von ihm repräsentierten Staat, und die Grenzen dieser Loyalität waren verträglich festgelegt. [BEM-WIŚNIEWSKA: 1998]

Das Bürgertum war in vielen Städten überwiegend deutsch, im Osten, wo bis auf Lemberg und Wilna Kleinstädte überwogen, jüdisch. Adam Krzemiński [KRZEMIŃSKI: 1993: 14] führt ein prägnantes Beispiel dafür ein, wie eine solche, mehrschichtige Identität um das Jahr 1620 bezeichnet werden konnte: „Canonicus cracoviensis, natione Polonus, gente Ruthenus, origine Judaeus" (Krakauer Kanonikus polnischer Nationalität, gebürtiger Ruthene jüdischer Herkunft) Vermutlich war Deutsch im Mund jenes Kanonikus die erste Kommunkationssprache in seinem Krakauer Alltag.

Das prominenteste Beispiel dafür, wie der im Modernisierungsprozess entstandene ethnische Nationalismus unseren Blick auf die vormoderne Realität versperrte, ist der deutsch-polnische Streit um die nationale Identität von Nicolaus Kopernikus. Die Familie Kopernikus stammte aus dem schlesischen Dorf Koperniki im Kreis Ottmachau. Der Vater von Nicolaus Kopernikus zog nach Thorn und dort wurde der berühmte Astronom geboren. Seine Briefe verfasste der Gelehrte in Deutsch, seine wissenschaftlichen Werke dagegen in der lateinischen Sprache. Politisch engagierte sich der katholische Priester auf der Seite des polnischen Königs. An der Universität Bologna trug er sich mit der Bezeichnug "natione germanorum“ ein und an der Universität Padua lautete die entsprechende Formel: „natione polonorum“. Bis heute werden beide Eintragungen, die keinesfalls als nationale Identifikationen im modernen Sinn zu verstehen sind, von den polnischen und deutschen Historikern im Streit um das vermeintliche - und auschliessliche - Polentum bzw. Deutschtum des grossen Astronoms gern als Beweise bemüht.

Derartige, auf eine eindeutige Zuschreibung zielende Argumentationslinien orientieren sich nur mehr an dem für die westeuropäischen Länder charakterisitischen Verlauf der Nationsbildung. Die nationale Identität, wie sie durch die Modernisierungsprozesse in westeuropäischen Staaten als deren untentbehrlicher Integrationsfaktor hervorbracht wurde, war vom Konzept einer ethnischen Homogenität geprägt. In ihrem Zentrum standen eine von der staatlichen Gewalt autorisierte Muttersprache neben dem Konzept vom Zusammenfliessen der territorialen, konfessionellen und sprachlichen mit den Staatsgrenzen. 

Massenideologie im ostmitteleuropäischen Raum verbreitete, war der polnische Staat von der Karte Europas längst verschwunden. Seine Bürger wurden in den drei Teilungsstaaten Preussen, Russland und Österreich mit unterschiedlichen Nationskonzepten und verschiedenen Entwicklungsstufen der Moderne konfrontiert. Ihrerseits entwickelten sie auch mannigfaltige Strategien für Identitätsverhandlungen. In den drei polnischen Teilgesellschaften entstanden viele neue Bewegungen und Konzepte. Andere Nationen meldeten ihrerseits Ansprüche auf eine künftige Staatlichkeit und es kam immer wieder zu Spannungen, in deren Zentrum ethnische Mobilisierung stand. In den von der Modernisierung weniger erfassten Gebieten konnten sich die vormodernen Identitätskonstrukte neben den aggressiven Ideologien allerdings bis zur Zeit der grossen Kriege im 20. Jahrhundert behaupten. nicht absolutistisch regierten Staates einer politischen Modernisierung im Sinne von Entwicklung der demokratischen Strukturen ein vorläufiges Ende setzte und die Entstehung des imperialen Machtsystems mit einer Vorrangstellung Preussens und Russlands möglich machte. So nimmt es nicht wunder, dass die neuen Bürger in diesen beiden Staaten als ein Sicherheitsrisiko betrachtet und mit der Zeit einer Zwangsassimillierung ausgesetzt wurden. [JAWORSKI u.a.: 2000; MÜLLER: 2005] problemlos zurecht zu kommen. Sie erinnerten sich noch an die Herrschaft der sächsischen Könige auf dem polnischen Thron und an Preussen, das in der nahen Vergangenheit noch ein Bestandteil des polnischen Staates war. Sie empfanden sich oft als preussische Untertanen katholischen Glaubens und der polnischen Muttersprache. Erst der Bismarcksche Kulturkampf rief auf der polnischen Seite eine ethnische Mobilisierung hervor. Die polnischen Intellektuellen, Lehrer, Repräsentanten der freien Berufe, vor allem aus Grosspolen, schufen in ihrer engagierten Arbeit Grundlagen die für polnische nationale Bewegung auch in Ermland oder Oberschlesien.

polnischen, politischen Exilzentren in Paris, in Italien und Amerika stellten zunächst für das zaristische Russland, in dem in Folge eines akut gewordenen Konfliktpotentials immer wieder polnische Aufstände ausbrachen, eine grosse Gefahr dar. Dort, im politischen Exil, entstand auch die polnische, romantische Nationalliteratur, in der ein wichtiges, u.a. an Novalis und Eichendorff geschultes Narrativ des 20. Jahrhunderts vorweggenommen wurde: Deprivation in Folge eines Heimatverlustes.

In Räumen, wo die beiden Sprachen: Polnisch und Deutsch nebeneinader existierten, in den späteren Vertreibungsgebieten: Ost- und Westpreussen, Ermland, Masuren, im Posenschen, in Oberschlesien oder Galizien wurde in jener Zeit Literatur in mehreren Sprachen publiziert. Eine Polikulturalität kennzeichnete das Leben in jenen Regionen. Auch die dort lebenden Polen haben sich um eine bilinguale Literatur bemüht, um nur zwei prominenteste Vertreter aus der Zeit der Jahrhundertwende, Stanisław Przybyszewski und Tadeusz Rittner, zu nennen, die ihre Schriften abwechselnd in Polnisch und in Deutsch verfassten.

Die rasche Industrialisierung brachte seit 1870 grosse Migrationswellen in und nach Deutschland. So kamen auch zahlreiche Polen ins Ruhrgebiet. Polnische Migranten waren bereits in der Zeit der polnisch-sächsischen Personalunion (Dresden und Leipzig) im deutschsprachigen Raum anwesend. Später strömten nach Westeuropa 
immer neue Migrationswellen nach den polnischen Aufständen, die im russisch besetzten Teil Polens blutig niedergeschlagen wurden. Mit der Zeit bildeten die Polen die zahlenmässig stärkste Migrationsgruppe im Deutschen Reich.

1871 lebten im neu vereinigten deutschen Staat ca 2,2 Millionen Polen (etwa 5,2\% der Gesamtbevölkerung), davon 500000 allein im Ruhrgebiet. Die Ruhrpolen integrierten sich relativ schnell. Ihr soziales Leben inmitten der deutschsprachigen Mehrheit stützten sie auf Pfarrgemeinden der katholischen Kirche (die ja sonst überall im dreigeteilten Polen auch viele Funktionen des ausbleibenden polnischen Staates übernahm). Daneben gründeten sie zahlreiche Polonia-Verbände, welchen die Aufgabe der Bildung und Pflege der nationalen polnischen Kultur zukam. Diese Strukturen überdauerten in der BRD bis heute. [SZULCZYŃSKI: 1999; KLESSMANN: 1978]

Zwischen den beiden Weltkriegen lebten in Deutschland rund 1,2 Millionen Bürger, die ihre Bindung an die polnische Sprache und Kultur deklarierten. Während der faschistischen Besatzung Polens kamen noch zwischen 3 und 4 Millionen Menschen als Zwangsarbeiter hinzu; die meisten kehrten nach dem Krieg in ihre Heimat zurück.

Nach den Erhebungen von Alexander ZajĄc [ZAJĄC: 2002] lebten in Deutschland gegen das Ende des 20. Jahrhundert über 1,5 Millionen Menschen polnischer Abstammung. Christoph Pallaske dagegen [PALLASKE: 2002] schätzt die Gruppe der polnischen Migranten auf rund 1 Million Menschen. Der rechtliche Status der polnischen Migranten ist nicht immer klar und er wird in vielen Fällen von den beiden Staaten unterschiedlich aufgefasst. Viele Menschen besitzen zwei Pässe, obwohl weder das polnische, noch das deutsche Gesetz eine doppelte Staatsangehörigkeit vorsehen.

Etwa 80000 Menschen, die Nachkommen der Wirtschaftsmigranten aus der Zeit noch vor dem Zweiten Weltkrieg sind, bekennen sich heute noch zu der Bindung an die polnische Sprache und Kultur. Ca. 50000 Menschen zählt die Gruppe der ehemaligen displaced persons. So waren Menschen genannt, die sich in Folge von Kriegshandlungen meist unfreiwillig in Deutschland als Zwangsarbeiter, KZ-Häftlinge oder Kriegsgefangene einfanden und dort geblieben sind. Eine zahlenmässig starke Gruppe, mehr als 250000 Menschen, bilden Migranten der Solidarność-Bewegung, bei denen auch wirtschaftliche Gründe kein seltenes Motiv bei der Ausreise darstellten. Die meisten davon siedelten in Deutschland in der Zeit zwischen 1987-1990. Ihr Verhältnis $\mathrm{zu}$ der alt eingesessenen Polonia war und ist oft distanziert.

32 In der Zeit zwischen 1950 und 1985 kamen nach Deutschland nach den polnischen Schätzungen über 850000 die sog. Spätaussiedler, polnische Bürger also, die sich zu der deutschen Abstammung bekannten und als deutsche Heimatvertriebene das kommunistische Polen verlassen durften. Viele von ihnen haben den Kontakt zu Polen aber nicht ganz abgebrochen, viele besitzen die rechtlich unzulässige, aber von den beiden Staaten gedultete doppelte Staatsangehörigkeit. Manche besuchen polnische Messen in der katholischen Kirche.

Im Statistischen Jahrbuch für die Bundesrepublik Deutschland 2000 sind dagegen andere Zahlen zu finden. Nach den deutschen Statistiken besassen lediglich 292000 Personen, also rund $4 \%$ der in Deutschland lebenden Ausländer, einen polnischen Pass.

Wenn man die Schätzungen des polnischen Soziologen ZajĄc annimmt, dann bilden die Polen in Deutschland unter den rund 170 dort lebenden Minoritäten die zweitgrösste Migrantengruppe (nach den Türken). Nach den deutschen Schätzungen wiederum kommt ihnen erst der fünfte Platz zu, nach Türken, Jugoslawen, Italienern und 
Griechen. Die Polen in Deutschland haben nach dem Zweiten Weltkrieg auch ihren juristischen Status einer nationalen Minderheit verloren.

Die deutsche Minderheit in Polen stellte für den 1918 wieder errichteten polnischen Staat ein kompliziertes Erbe dar. Aus den drei Teilungsstaaten: Russland, Deutschland und Österreich kommend, waren die Deutschen in Polen alles andere als eine homogene Gruppe. Ein anderer wichtiger Umstand war, dass die allgemeine Stimmung im Lande mehr von Konflikten zwischen mannigfaltigen Minoritäten geprägt wurde, als von Formen eines friedlichen Zusammenlebens.

Die Stärke der deutschen Minderheit in Polen wurde an Hand der Volkszählungen auf etwa 1, 1 Millionen Menschen geschätzt. Nach 1931 schrumpfte sie auf ca. 784000 Personen. [TOMASZEWSKI: 1991] Die polnische Bevölkerung sah in den polnischen Deutschen oft ein trojanisches Pferd, von dem aus eine Bedrohung für die Unabhängigkeit Polens ausgehen könnte. Man sprach von der Fünften Kolonne. Neben den loyalen Staatsbürgern befanden sich unter den polnischen Deutschen in der Tat glühende Anhänger Hitlers. Auch wenn sie für die polnischen Deutschen nicht repräsentativ waren, so zogen sie doch die Aufmerksamkeit der Öffentlichkeit auf sich.

Der Krieg und die Besatzung verschärften nur noch die bestehenden Konflikte. Die Nazis haben eine vierstufige „Deutsche Volksliste“ eingeführt. Wer sie unterschrieb, genoss verschiedene Privilegien, galt aber in den polnischen Augen als Verräter. Wer sie nicht unterschrieb, der wurde von den Nazis verschiedenen Repressalien ausgesetzt.

38 So nimmt es nicht wunder, dass die nach dem Krieg durchgeführte Zwangsaussiedlung der Deutschen aus dem polnischen Staat bei der polnischen Bevölkerung einhellige Unterstützung fand. Auf Grund des Potsdamer Abkommens kamen an Polen mehrere Provinzen des ehemaligen deutschen Ostens. Polen musste im gleichen Zug seine östlichen Regionen an die Sowjetunion abtreten. Die Zwangsmonoethnisierung der betreffenden Gebiete dauerte etliche Jahre und bedeutete eine Umsiedlung von Millionen Menschen.

In mehreren Etappen wurde zwischen 1945 und 1948 eine Verifizierungsaktion durchgeführt, deren offizielles Ziel war, auf dem Weg einer Repolonisierung die zuvor stattgefundene Germanisierung der einheimischen Bevölkerung rückgängig $\mathrm{zu}$ machen. Diejenigen, die im Lande bleiben wollten, mussten schriftlich um die polnische Volks- und Staatszugehörigkeit ansuchen und nicht selten in Berufung gehen. Der Prozentsatz der abgelehnten Anträge lag unter 5\%, in manchen Kreisen um 20\%. Nicht allen Menschen wurde das Optionsrecht zuerkannt. Man teilte die Bevölkerung nach den von den polnischen Experten erarbeiteten Kriterien in die ethnischen Deutschen und die sog. autochthone Bevölkerung. Im Rahmen der Zwangsaussiedlung wurden in den Jahren 1945-1950 nach den polnischen Schätzungen 3,2 Millionen Deutsche hinter die westliche Grenze deportiert. Die deutschen Schätzungen gehen von über 4,5 Millionen Heimatvertriebenen aus (bei der Gesamtzahl von 12,5 Millionen aller aus Ostmitteleuropa vertriebenen Deutschen).

In Niederschlesien, besonders in Breslau/Wrocław und Waldenburg, wurde ein Teil der als ethnische Deutschen eingestuften Menschen im Lande zurückgelassen, um die Industrie instand $\mathrm{zu}$ setzen oder zu halten. Bis 1956 erschien für diese Gruppe in Breslau ein Wochenblatt, betitelt „Die Arbeiterzeitung“. Die Deutschen genossen kirchliche Fürsorge und bis 1961 hatten sie in Breslau und Liegnitz auch ihre Schulen. Anfang der 50er Jahre wurde ihnen die polnische Staatsangehörigkeit oder Ausreise in 
die DDR angeboten. Diejenigen, die eine solche Lösung ablehnten, galten als staatenlos. 1957 durften sie die Deutsche Soziokulturelle Gesellschaft gründen, die einzige, offiziell anerkannte Organisation der deutschen Minderheit in Polen bis 1989.

Nach 1956 wendete sich das Blatt. Viele vom stalinistischen Terror enttäuschte und vom bundesdeutschen Wirtschaftswunder angezogene Menschen, die vorher die polnische Staatsangehörigkeit angenommen hatten, zeigten sich nun als ausreisewillig. Über 30 Jahre lang dauerte der polnisch-bundesdeutsche Streit um die Anerkennung der ausreisewilligen Menschen als Deutsche. Die Auswanderungswellen folgten in mehreren Etappen: 1957-1961; 1970-1978; 1987-1990.

Nach 1989 passte die Republik Polen ihre Gesetze an die europäischen Standards an. In unterschiedlichen Organisationsformen der deutschen Minderheit in Polen (wobei die Spannung zwischen Kattowitz und Oppeln sich in jenen Strukturen besonders abzeichnet), die ihre parlamentarische Repräsentanz besizt und auch in Kommunalwahlen Erfolge erzielt, gruppieren sich derzeit ca 300000 Menschen. In der letzten Volkszählung 2004 gaben allerdings 172000 Personen, die sich vorher als Deutsche identifiziert hatten, nun GórnoślĄzak/Oberschlesier als Bezeichnung für ihre nationale Identität an. Manche von ihnen wollten in Strassburg Anerkennung der Oberschlesier als eine separate Nation durchsetzen oder zumindest den Status einer ethnischen Minderheit für oberschlesische Bevölkerung erzielen.

Die deutsche Minderheit in Polen verfügt derzeit über eigene Medien, wie Hörfunk, Zeitung und Fernsehprogramme. Ihre wichtigste Zeitung, "Schlesisches Wochenblatt", wird vom Oppelner Journalisten, Engelbert Miś, der früher für die katholischen Blätter schrieb, redigiert. Sie ist zur Jahreswende 1994/95 entstanden.

Anfang 1994 entstand die Gesellschaft deutscher Autoren in Polen, aber von einer Literatur der deutschen Minderheit in Polen zu reden, ist wohl noch vorzeitig. So zumindest urteilt Erhard Bastek, ein Autor und Übersetzer aus Bytom/Beuthen, der die Gesellschaft ins Leben gerufen hat. In der 1998 entstandenen Monatsschrift "Oberschlesisches Bulletin“ werden literarische Texte publiziert; bislang erschienen fast 60 Hefte. Zu den mit der Zeitschrift verbundenen Autoren gehören neben Erhard Bastek u.a. auch Anna Makselon, die Rundfunkjournalistin Ingeborg Odelga und der Lyriker Zbigniew Stadler-Żelisławski. Das erste edierte Buch trug den Titel „Gedichte in der Heimat - Wiersze stron rodzinnych" und war eine Textanthologie von 11 Autoren.

Der schlesischen Literatur und Kultur widmet sich auch das nach 1989 gegründete Joseph von Eichendorff-Konversatorium, ein 35 Mitglieder zählender Verein, der sich als eine Fortsetzung der bis 1944 tätigen Oppelner Eichendorff-Gemeinde versteht und eine Vierteljahreszeitschrift unter dem gleichnamigen Titel herausgibt, in der auch literarische Texte erscheinen. Eine wichtige Rolle spielt das 1998 gegründete Haus der Deutsch-Polnischen Zusammenarbeit mit dem Sitz in Gleiwitz. Träger des Vereins sind zahlreiche Stiftungen und Institutionen, u.a. Verband der Sozial-kulturellen Gesellschaften der Deutschen in Polen, Landesregierung Nordrhein-Westfalen, Polnischer Deutschlehrerverband, Regionale Wirtschaftskammer und mehrere deutsche Stiftungen. Eins der Ziele des Hauses ist die Förderung der freundschaftlichen deutschpolnischen Beziehungen in Zusammenarbeit mit den Organisationen und Institutionen der deutschen Minderheit in Polen und Förderung der deutschen Minderheit in ihrem Dialog mit der polnischen Mehrheit. [KALCZYŃSKA:2004] 

über eigene Zeitungen und Verlage, deren Ziel war, polnische Sprache und Kultur zu pflegen, sowie eine Verbindung $\mathrm{zu}$ der katholischen Kirche und Religion aufrechtzuerhalten. Viele Priester waren in jenem Umkreis tätig. Diese Tradition wurde nach dem Zweiten Weltkrieg fortgesetzt. Zahlreiche Intellektuellen, Journalisten und Schriftsteller kamen in mehreren Migrationswellen als politische Exulanten nach Deutschland. In grossen Städten schufen sie eigene Kulturzentren, mit polnischen Zeitungen, Zeitschriften und auch Buchverlagen. Die meisten Publikationen waren freilich in der polnischen Sprache verfasst und an das polnischsprachige Lesepublikum in Deutschland gerichtet. Berlin, Hamburg, Bremen, Stuttgart und München waren nicht die einzigen Schauplätze dieser als eine Initiative von unten spontan entwickelten Kultur. In den 80er Jahren radikalisierten die politischen SolidarnośćExulanten das polnische kulturelle Leben in Deutschland. Viele unter ihnen waren Schriftsteller, die sich in Polen bereits einen Namen gemacht haben und nun von ihrem Publikum abgetrennt wurden. Mit der Zeit wandten sie sich immer mehr den Belangen ihrer neuen Heimat zu. Manche gingen über die Sprachgrenze hinaus und schrieben in Deutsch.

Die polnische Migrantenliteratur in Deutschland stellt ein kaum erforschtes Gebiet dar. Dort, wo sie die Aufmerksamkeit der Medien auf sich zu ziehen vermochten, sind polnische Migranten, wie der Berliner Klub der Polnischen Versager oder der Künstlerverein WIR, einiger-maßen bekannt. Andere veröffentlichen in kleinen Verlagen oder agieren in kleineren Kreisen. Einige, wie Dariusz Muszer oder Artur Becker, sind bereits mit literarischen Stipendien und Preisen ausgezeichnet.

t Autoren unter ihnen, die in Polen deutsche nationale Identität entwickelten, wie Britta Wuttke, Peter Lachmann oder Artur Becker. Die erste Sprache Wuttkes war Deutsch, ihr Erstling, betitelt „Homunculus z tryptyku“ [dt.: „Homunculus aus dem Triptychon“] verfasste die Medizinerin 1977 allerdings in Polnisch. Später durfte sie ausreisen und lebt derzeit in Berlin, wo sie Kontakte zu WIR unterhält. Artur Becker, geboren 1968 in Bartoszyce (Ermland) lernte richtiges Deutsch erst nach seiner 1985 erfolgten Migration nach Deutschland. Er schreibt in Deutsch, ein wichtiger Schauplatz seiner Erzählungen, Gedichte und Romane bleiben das masurische Bartoszyce und die Gegend um den Dadajsee. Peter Lachmann lernte Polnisch als kleines Kind im polnisch gewordenen Gleiwitz. Er lebt als Theatermacher, Übersetzer und Autor in Polen und in Deutschland und schreibt in den beiden Sprachen.

50 Andere Schriftsteller, wie Janusz Rudnicki oder Natasza Goerke, die in den 80er Jahren emigrierten und derzeit in Deutschland leben, machten ihre Existenz in einem transitorischen Raum zum wichtigsten Thema ihrer in Polnisch verfassten Werke. Rudnickis „Listy z Hamburga“ [Briefe aus Hamburg] und Goerkes humorvolle und artifizielle Kurzgeschichten sind von der polnischen literarischen Kritik als represäntative Texte in den Kanon der modernen polnischen Prosa aufgenommen. Der 1959 in Grosspolen geborene Lyriker und Prosaist Dariusz Muszer dagegen hat sich nach einem gelungenen lyrischen Debüt und mehreren Publikationen in Polen schliesslich doch für Deutsch als seine Literatursprache entschieden. Er hat am literarischen Leben sowohl in Deutschland wie auch in Polen einen regen Anteil. In seinem Roman „Die Freiheit riecht nach Vanille“ thematisierte er auf eine provokante Weise Aporien der nationalen Identitätszuschreibung durch die Mächtigen. 
51 grosse Gruppe von Kulturschaffenden bilden, sind in ihrer Tätigkeit der Öffentlichkeit in Deutschland wenig bekannt. Auch ihr Werk fristet im deutschen Kulturleben gleichsam ein Nischendasein. Ein Grund dafür mag wohl auch darin liegen, dass eine solche Migration sich in die gängigen Muster des Sprechens über die Transkulturalität wie die der politischen Korrektheit schlecht einschreibt.

\section{BIBLIOGRAPHIE}

Interkulturelle Literatur in Deutschland: ein Handbuch. Hg. von Carmine Chielino. Stuttgart; Weimar: Metzler, 2000, insb. S.195-196

Gabriel Laub: Skusenosti, Prag 1967

Kalczyńska, Maria: Polsko-niemiecki leksykon biograficzny. Polnisch-deutsches biographisches Lexikon. Opole 2001

Ewa Bem-Wiśniewska: Funkcjonowanie nazwy Polska w języku czasów nowożytnych (1530-1795). Warszawa: Wydawnictwo DIG 1998

Adam Krzemiński: Polen im 20. Jahrhundert. Ein historischer Essay, München: Beck 1993

Rudolf Jaworski/Christian Lübke/Michael G. Müller: Eine kleine Geschichte Polens, Frankfurt/ M.: 2000

Michael G. Müller: Rozbiory Polski. Historia Polski i Europy XVIII wieku. Tłum.: Monika WrzosekMüller. Poznań: PTPN 2005

Andrzej Szulczyński: Zarys dziejów Polonii niemieckiej, Berlin 1999;

Christian Klessmann: Polnische Bergarbeiter im Ruhrgebiet 1870-1945, Göttingen 1978

Alexander ZajĄc: Sytuacja Polaków w Niemczech. (In:): Maria Masłowska (Hg.:) Polonia w Niemczech. Teil, Warszawa 2002

Christoph Pallaske: Migration aus Polen in die Bundesrepublik Deutschland in den 1980er und 1990er Jahren. Münster 2002

Jerzy Tomaszewski: Mniejszości narodowe w Polsce XX w., Warszawa 1991 Jerzy Tomaszewski: Deutsche in Polen. (in:) Ewa Kobylińska, Andreas Lawety und Rüdiger Stephan (Hag.:) Deutsche und Polen. 100 Schlüsselbegriffe. München 1992, S. 500-508

Kalczyńska, Maria: Kultura ksiĄżki polskiej w Niemczech. Katowice 2004

\section{RÉSUMÉS}

Das Wissen um polnische Migranten in Deutschland und die deutsche Minderheit in Polen ist von einer beschränkten Wahrnehmung geprägt. Der Kritiker Marcel Reich-Ranicki, der Übersetzer und Literturwissenschaftler Karl Dedecius oder der Satiriker Gabriel Laub, werden teilweise nicht als polnischstämmig wahrgenommen. Sie kommen aus polnischen Regionen, welche vor der 
polnischen Staatsgründung Mischräume waren, in welchen Deutsch als Kommunikationssprache diente und sind Teil jener großen polnischen Migrationswellen nach Deutschland, die sich ab 1870 regelmäßig wiederholten.

Trotz der Repolonisiserungspolitik und trotz der Zwangsaussiedlung nach dem Zweiten Weltkrieg mußte ein Teil der als Deutsche eingestuften Bevölkerung in Oberschlesien bleiben. Sie wurde vor allem zur Instandsetzung der maroden Industrie zurückgehalten. Obwohl ab 1956 viele Deutsche aus Polen ausgereist sind, zählt die verbleibende deutsche Minderheit derzeit noch etwa 174000 Mitglieder, die über ihre eigenen Kultureinrichtungen verfügen und ihre eigene Literatur haben. Leider sind deren Vertreter wie Britta Wuttke, Peter Lachmann, Dariusz Muser oder Artur Becker in Deutschland kaum bekannt.

L'existence d'une émigration polonaise en Allemagne et d'une minorité allemande en Pologne est mal connue de part et d'autre. Que ce soit Marcel Reich-Ranicki, critique littéraire, Karl Dedecius, traducteur et chercheur en littérature ou bien encore Gabriel Laub, auteur satirique, ils ne sont, pour la plupart ,pas perçus, comme étant d'origine polonaise. Ils sont originaires de ces régions de Pologne où, avant la création d'un Etat polonais, on trouvait des populations mélangées, et où l'allemand servait de langue de communication; ils font partie de cette grande vague d'émigration polonaise vers l'Allemagne à laquelle on assista à partir de 1870. Malgré la politique de repolonisation et malgré l'exil forcé d'un certain nombre d'entre eux au lendemain de la Seconde Guerre Mondiale, une partie de la population considérée comme allemande dut rester en Haute-Silésie. Elle fut retenue avant tout dans le but de remettre en état de fonctionnement une industrie en piteux état. Bien qu'à partir de 1956 beaucoup d'Allemands aient émigré de Pologne, la minorité allemande restante s'élève encore à ce jour à environ 174000 personnes qui disposent de leurs institutions culturelles et de leur littérature propres. Malheureusement, leurs représentants tels que Britta Wuttke, Peter Lachmann, Darius Muser ou Artur Becker sont très peu connus en Allemagne.

INDEX

Mots-clés : Pologne, émigration polonaise, minorité allemande

\section{AUTEURS}

\section{ELŻBIETA KATARZYNA DZIKOWSKA}

Universität Wrocław 